\title{
Ethnicity and Environment May Affect the Phenotype of Immune Thrombocytopenic Purpura in Children
}

\author{
THOMAS KÜHNE, WILLI BERCHTOLD, TRAN VAN BE, TRAN VAN BINH, AND PAUL IMBACH \\ Division of Oncology/Hematology [T.K., P.I.], University Children's Hospital Basel, Basel, Switzerland; \\ University of Applied Sciences of Aargau [W.B.], Brugg, Windisch, Switzerland; and Blood Transfusion \\ and Hematology Center [T.H.Be, T.H.Binh], District 5, Ho Chi Minh City, Vietnam
}

\begin{abstract}
Little is known about the influence of environmental and ethnic factors on the epidemiology of immune thrombocytopenic purpura (ITP). Therefore we compared the initial presentation and condition after 6 mo in 90 Vietnamese and 89 German and Swiss children with newly diagnosed ITP. Data from the two cohorts were collected within the same time period. No differences in age and sex were observed between the Asian and European cohorts, but significant differences between initial platelet count, the occurrence of dry versus wet bleeding symptoms, and infection preceding the onset of ITP were found. Children who had chronic ITP also differed with respect to
\end{abstract}

\section{ABSTRACT}

platelet count and postinfectious state, but not initial bleeding type. In addition, chronic ITP occurred more often than expected with a male to female ratio of 1.2 in Vietnam and 2 in Germany and Switzerland. The data support the potential influence of environmental or ethnic factors on the different aspects of ITP, and point to the need for further epidemiologic investigations.

(Pediatr Res 48: 374-379, 2000)

\section{Abbreviations}

ITP, immune thrombocytopenic purpura

IVIg, i.v. immunoglobulins
ITP is a bleeding disorder characterized by antibodymediated platelet destruction in the monocytic phagocytic system resulting in thrombocytopenia, defined as a platelet count $<150 \times 10^{9} /$ L. Acute ITP typically occurs in previously healthy children between the age of 2 to $6 \mathrm{y}$ and is usually a short and self-limited disease independent of initial treatment $(1,2)$. Many children with acute ITP have a history of an infectious illness, typically a viral upper respiratory tract infection, or an immunization in the weeks preceding the onset of purpura (3-6). Chronic ITP is arbitrarily defined as thrombocytopenia persisting for $>6 \mathrm{mo}$ and is rare in childhood, occurring in approximately $20 \%$ of children with ITP (7). To date, no predictors of chronic ITP have been identified in children, although many investigators believe that female sex, age $>10 \mathrm{y}$, a relatively high platelet count at presentation $\left(>20 \times 10^{9} / \mathrm{L}\right)$, and an insidious onset of symptoms with signs of bleeding for $>2$ to $4 \mathrm{wk}$ before diagnosis are associated with the chronic form of the disorder (7-9).

According to the practice guidelines of the American Society of Hematology, clinical management is often based on uncontrolled case series of selected patients with outcome

Received November 16, 1999; accepted May 12, 2000.

Correspondence and reprint requests: Thomas Kühne, M.D., University Children's Hospital Basel, Division of Oncology/Hematology, Römergasse 8, Postfach, CH-4005 Basel, Switzerland. evaluation using only platelet counts, and on personal expert opinion $(7,10)$. Epidemiologic aspects are also reflected by many "dogmas" based on insufficient data or on personal expert opinion. Whether ITP represents a disorder with heterogeneous pathophysiologic characteristics influenced by environmental or ethnic factors is unclear. The aim of the present study was to compare the clinical data of two similar but ethnically different cohorts of children seen consecutively with newly diagnosed ITP from the time of first presentation until 6 mo, at which time the diagnosis of acute or chronic ITP was established. It should be emphasized that the value of this study lies in the comparison of the data between the two different cohorts observed within the same time period.

\section{METHODS}

Participating centers. The hematology reference center for Ho Chi Minh City, Vietnam, and 14 pediatric centers, 12 in German-speaking Switzerland and 2 in southern Germany close to Switzerland, participated. The population served by the Vietnamese and the European centers was estimated to be 11 and 8 million inhabitants, respectively. A designated pediatric hematologist at each center was responsible for the collection and transfer of data to the study center. The data were processed anonymously. Children were enrolled in the study during a 2-y-period. Informed consent was obtained, and the 
study was approved by the Ethical Committee of the University Children's Hospital Basel.

Diagnosis. Children aged $<19 \mathrm{y}$, in whom the diagnosis of ITP was established at each center, were included in the study. Inclusion criteria were a platelet count of $<150 \times 10^{9} / \mathrm{L}$, with a normal blood smear and no secondary causes for ITP, such as HIV infection, systemic lupus erythematosus, and lymphoproliferative disorders. The finding of normal or increased megakaryocytes in a bone marrow aspirate was an optional factor. In all centers, platelet measurements $<50 \times 10^{9} / \mathrm{L}$ were confirmed by manual determination, using a Neubauer or Spencer counting chamber after hemolysis and clear light microscopy. Dry hemorrhage was defined as the presence of petechiae, ecchymoses, or suffusions of the skin, and wet hemorrhage as mucous membrane bleeding, such as epistaxis, gingival bleeding, hematuria, menorrhagia, or gastrointestinal hemorrhage. The occurrence of intracranial hemorrhage within the first 6 mo was specifically documented. Other bleeding disorders, such as von Willebrand disease, or platelet function abnormalities were not routinely screened. Infection was defined as fever or symptoms involving the upper respiratory tract, ear, nose, or gastrointestinal tract, or any specific infection or immunization. Infections were classified according to whether they occurred within 14,21 , or $>21 \mathrm{~d}$ before the diagnosis of ITP. Chronic ITP was defined as a persistence of thrombocytopenia (platelet count $<150 \times 10^{9} / \mathrm{L}$ ) for $>6 \mathrm{mo}$.

Data collection. Two comprehensive clinical data forms were completed for each child, the first at the time of the initial diagnosis of ITP, and the second 6 mo later, at which time the classification of ITP as chronic was able to be made. The first form included patient age, sex, platelet count at diagnosis, prior infection, bone marrow aspiration if performed, treatment, and details on hospitalization. The second form recorded information during the 6-mo follow-up period, including platelet count, bleeding, initial therapy, additional treatment, and total time of hospitalization.

Treatment. Treatment included IVIg, corticosteroids, or a combination of IVIg plus corticosteroids. Some patients received no treatment. Dosages and treatment duration were not taken into account. The type of treatment administered at the time of diagnosis and treatment during the 6-mo observation period were recorded separately.

Statistical analysis. The main objective was to detect differences between the two cohorts of patients from Vietnam and from Germany and Switzerland, which necessitates a twosample statistical analysis. Therefore, both the $t$ test and MannWhitney $U$ test were used for the comparison of all continuous data. Logarithms for the $t$ test were used for abnormal data, e.g. initial platelet counts. Both methods produced similar results, with some differences in the $p$ values. In this paper, only the $p$ values obtained by the Mann-Whitney $U$ test are reported. Contingency tables with dimensions $2 \times 2$ to $2 \times 4$ were used to analyze data classified into several categories. The $\chi^{2}$ test and Fisher's exact test were performed for all comparisons, but only the $p$ values derived from the Fisher's exact test are reported. Calculations were performed using STATA software (Release 5.0, Stata Corporation, College Station, TX, U.S.A.) and StatXact4 (CYTEL Software Corporation, Cambridge, MA, U.S.A.) for the exact test in large tables.

\section{RESULTS}

One hundred seventy-nine consecutively seen children, 90 from Vietnam and 89 from Germany and Switzerland, were included in the study. Eighty-two (91.1\%) of the 90 Vietnamese, and $71(81.6 \%)$ of the 89 German and Swiss children were eligible for analysis. Ineligibility was because of unavailability of information on follow-up 6 mo after the initial diagnosis. The clinical and laboratory analyses at initial presentation are summarized in Table 1. The age distribution of German and Swiss children showed a maximum at $4 \mathrm{y}$, whereas in Vietnam two peaks, one during infancy and another in adolescence, were seen with a trough between 2 and 8 y of age (Fig. 1). Subgroup analysis of different age groups $(<2,2-4,4-10$, and $>10 \mathrm{y}$ ), using contingency tables, $\chi^{2}$, and Fisher's exact test, revealed younger children in the age group $<2$ y $(p<0.038)$, and older children in the age group $>10$ y $(p<0.01)$ in the Vietnamese group of patients, compared with those from Germany and Switzerland. The difference in mean age between the two cohorts as a whole, however, was not statistically significant, as indicated by the Mann-Whitney $U$ test $(p=0.77)$. Comparing the two cohorts, the mean age of boys and of girls, and of children with acute and of those who developed chronic ITP was not statistically different (Table 1). Also the male to female ratio did not differ between the two cohorts. A slight predominance of boys over girls was observed both in the Vietnamese (61\%) and in the German and Swiss cohort (54\%; Table 1).

Platelet counts. The initial mean platelet count was statistically significantly higher in the Vietnamese than in the German and Swiss cohort $(p<0.001)$. Also, the distribution of the initial platelet count was more dispersed among Vietnamese patients than within the group of German and Swiss children (Fig. 2). Initial platelet counts of $<20 \times 10^{9} / \mathrm{L}$ were found in 50 Vietnamese children $(61.7 \%)$ and in 63 of the German and Swiss patients $(88.7 \%)$. This difference was statistically significant for boys $(p<0.001)$, but not for girls. In patients with acute ITP the initial platelet count showed a mean value of $23.9 \pm 21.5 \times 10^{9} / \mathrm{L}$ in the Vietnamese cohort, and of $9.6 \pm$ $13.6 \times 10^{9} / \mathrm{L}$ in the German and Swiss cohort $(p<0.001)$. Similarly, patients who developed chronic ITP showed a mean initial platelet count of $24.3 \pm 21.0 \times 10^{9} / \mathrm{L}$ in the Vietnamese cohort, and of $10.8 \pm 7.6 \times 10^{9} / \mathrm{L}$ in the German and Swiss cohort $(p=0.001)$. Thus, the initial mean platelet counts showed no differences between acute and chronic ITP in both cohorts. There was no difference in the prevalence of chronic ITP between the two cohorts being observed in $42.7 \%$ (35 of 82) of the Vietnamese patients, with 19 boys and 16 girls (male to female ratio of 1.2 ), and in $42.3 \%$ (30 of 71) of these from the German and Swiss group with 20 boys and 10 girls (male to female ratio of 2.0). The sex differences between the two cohorts were also not statistically significant. Increasing age did not influence the incidence of chronic ITP, children $>10 \mathrm{y}$ being equally distributed in both acute and chronic ITP groups with no difference between the two cohorts. An initial platelet 
Table 1. Clinical and laboratory characteristics of patients with acute ITP and those who subsequently were classified with chronic ITP at initial presentation

\begin{tabular}{|c|c|c|c|}
\hline & Vietnam & $\begin{array}{l}\text { Germany and } \\
\text { Switzerland }\end{array}$ & $p$ values \\
\hline$n$ & 82 & 71 & NS \\
\hline \multicolumn{4}{|l|}{ Sex } \\
\hline Acute ITP (M:F) & $31 / 16(66 \% / 34 \%)$ & $18 / 23(44 \% / 56 \%)$ & NS \\
\hline Chronic ITP (M:F) & $19 / 16(54 \% / 46 \%)$ & $20 / 10(67 \% / 33 \%)$ & NS \\
\hline \multicolumn{4}{|l|}{ Age* } \\
\hline Chronic ITP & $7.0 \pm 4.9(0.2-18.3)$ & $6.1 \pm 3.1(1.4-13.9)$ & NS \\
\hline Boys with acute ITP & $6.0 \pm 3.6(0.3-17.8)$ & $6.8 \pm 6.4(1.4-15.2)$ & NS \\
\hline Boys with chronic ITP & $5.9 \pm 4.5(0.8-15.5)$ & $6.1 \pm 4.9(2.0-13.9)$ & NS \\
\hline Girls with acute ITP & $7.5 \pm 5.1(0.3-17.3)$ & $5.1 \pm 4.2(0.4-10.2)$ & NS \\
\hline Girls with chronic ITP & $8.3 \pm 6.6(0.2-18.3)$ & $6.1 \pm 6.1(1.4-6.1)$ & NS \\
\hline Initial platelet count $\dagger$ & $24.1 \pm 21.2(2-98)$ & $10.1 \pm 11.4(1-68)$ & $<0.001$ \\
\hline \multicolumn{4}{|l|}{ Wet hemorrhage } \\
\hline Acute ITP & $20 / 45(44 \%)$ & $28 / 41(68 \%)$ & 0.031 \\
\hline Chronic ITP & $16 / 33(49 \%)$ & $15 / 30(50 \%)$ & NS \\
\hline Platelet count $\dagger$ & $20.4 \pm 20.1(2-98)$ & $6.3 \pm 5.5(1-23)$ & $<0.001$ \\
\hline Pre-ITP infection & $13 / 82(16 \%)$ & $47 / 71(66 \%)$ & $<0.001$ \\
\hline Acute ITP & $7 / 47(15 \%)$ & $28 / 41(68 \%)$ & $<0.001$ \\
\hline Chronic ITP & $6 / 35(17 \%)$ & $19 / 30(63 \%)$ & $<0.001$ \\
\hline
\end{tabular}

$*$ age in years: mean $\pm \mathrm{SD}$ (range), $\uparrow$ platelet count $\left(\times 10^{9} / \mathrm{L}\right)$ : mean $\pm \mathrm{SD}$ (range).

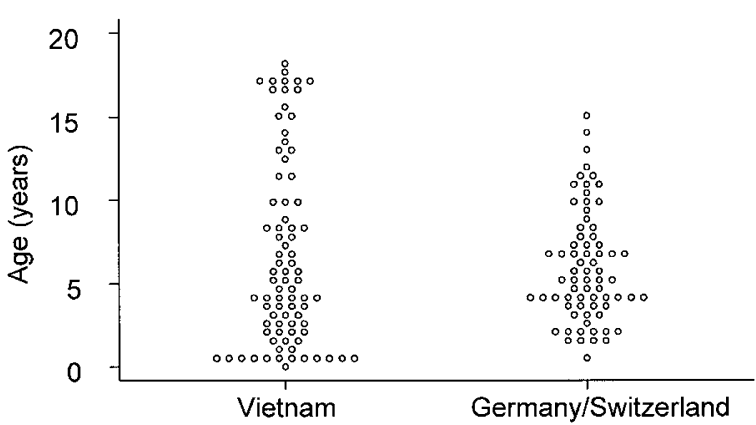

Figure 1. Age distribution of all children at the time when the initial diagnosis of ITP was made. The values of Vietnamese children are shown on the left and of Swiss and German children on the right.

count $>20 \times 10^{9} / \mathrm{L}$ was observed more frequently in Vietnamese patients $(n=31)$, than in the German and Swiss cohort $(n=8)$, although the proportion of children having chronic ITP was similar in both cohorts. Children with chronic ITP tended to exhibit a lower platelet count at 6 mo in Vietnam $\left(54.2 \pm 43.0 \times 10^{9} / \mathrm{L}\right)$ than in Germany and Switzerland $\left(74.2 \pm 41.0 \times 10^{9} / \mathrm{L} ; p=0.05\right.$; Fig. 3$)$, whereas platelet counts at 6 mo in children with acute ITP showed no significant statistical difference $\left(252.9 \pm 51.8 \times 10^{9} / \mathrm{L}\right.$ and $273.5 \pm$ $95.6 \times 10^{9} / \mathrm{L}$, respectively).

Bleeding. The frequency of bleeding signs at diagnosis was equal in both cohorts. However, a statistical difference was found in the type of bleeding, if all children were considered. Dry hemorrhage occurred more frequently in the Vietnamese cohort (42 of 78,54\%), whereas wet hemorrhage was observed more often in the German and Swiss patients (43 of 71, 61\%). This difference was only statistically significant in children

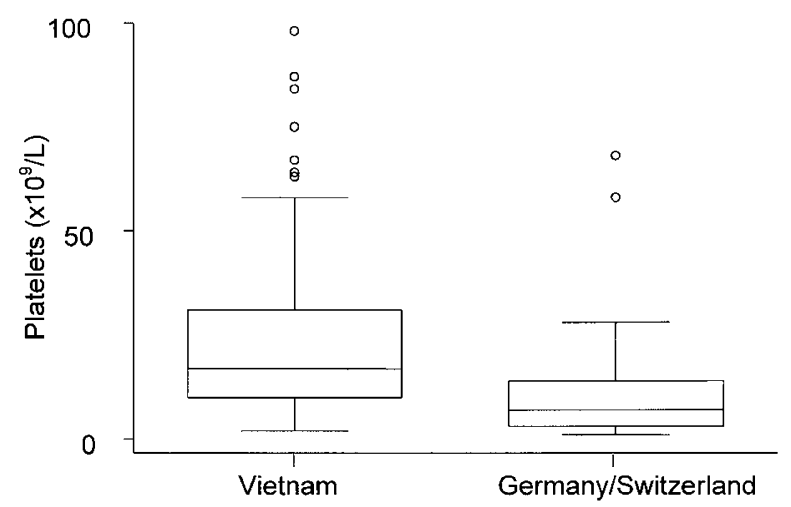

Figure 2. The platelet count of all children at the time when the initial diagnosis of ITP was made. The data are presented as box plots. The values of Vietnamese children are shown on the left and of Swiss and German children on the right.

with acute ITP with either dry or wet hemorrhage (Table 1). Considering patients with an initial platelet count of $<20 \times$ $10^{9} / \mathrm{L}$, there was no significant difference $(p=0.32)$ of the occurrence of dry and wet hemorrhage between the patients from Vietnam ( 21 and 25 of 46 children), and those from Germany and Switzerland (22 and 40 of 62 children). Eleven Vietnamese and two German and Swiss patients with wet hemorrhage revealed a platelet count of $>20 \times 10^{9} / \mathrm{L}$. The mean initial platelet count of Vietnamese children with dry hemorrhage was $28.6 \pm 22.2 \times 10^{9} / \mathrm{L}$ (range, $\left.3-87 \times 10^{9} / \mathrm{L}\right)$ and of the German and Swiss group, $15.9 \pm 15.2 \times 10^{9} / \mathrm{L}$ (range, $2-68 \times 10^{9} / \mathrm{L} ; p=0.003$ ). The patients with wet hemorrhage showed a mean platelet count of $20.4 \pm 20.1 \times$ $10^{9} / \mathrm{L}$ (range, $2-98 \times 10^{9} / \mathrm{L}$ ) in the Vietnamese cohort and of 


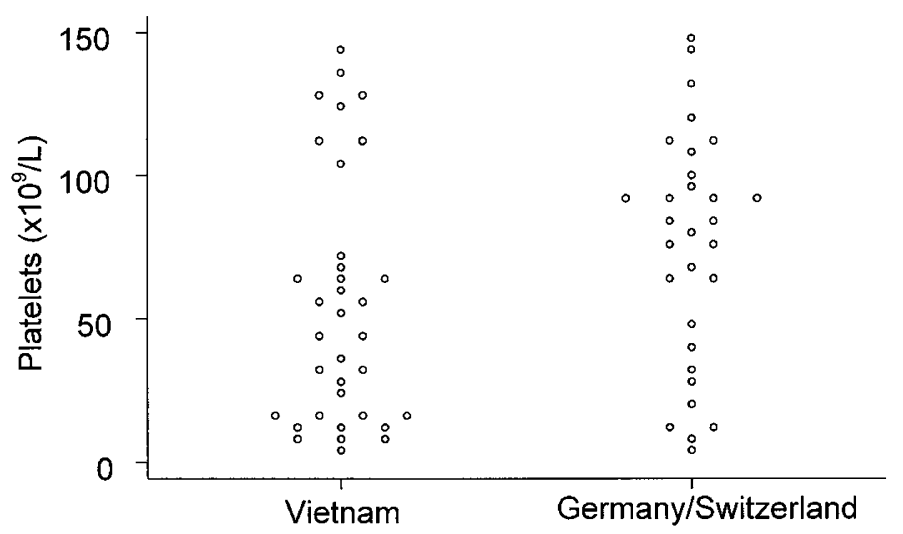

Figure 3. The platelet count of children with chronic ITP at 6 mo after the diagnosis of ITP. The values of Vietnamese children are shown on the left and of Swiss and German children on the right.

$6.3 \pm 5.5 \times 10^{9} / \mathrm{L}$ (range, $\left.1-23 \times 10^{9} / \mathrm{L}\right)$ in the German and Swiss cohort $(p<0.001)$. If age groups were analyzed $(<1 \mathrm{y}$, $1-5 \mathrm{y}$, and $>5 \mathrm{y})$, no difference was observed regarding bleeding frequency or type, neither among children within a specific cohort, nor when the two cohorts were compared with each other. No episodes of major hemorrhage requiring red blood cell transfusions occurred in either cohort, and none of the children suffered intracranial hemorrhage or died because of major hemorrhage within the 6-mo period of observation.

Infection. The frequency of pre-ITP infection was much higher within the German and Swiss cohort (47 of 71, 66\%) than in the Vietnamese group (13 of 82, 16\%), with high statistical significance $(p<0.001)$. However, the frequency of infection did not differ significantly between acute and chronic ITP within either cohort. Considering patients with an initial platelet count of $<20 \times 10^{9} / \mathrm{L}$, pre-ITP infection was seen in $9(18 \%)$ from the Vietnamese group, and in $40(65 \%)$ from the German and Swiss cohort $(p<0.001)$. Finally, whether the infection was diagnosed within 14,21 , or $>21 \mathrm{~d}$ preceding the onset of ITP had no influence on the various aspects of ITP studied.

Management. Bone marrow aspiration was performed more often in patients from Vietnam than in those from Germany and Switzerland (73\% versus $28 \%, p<0.0001)$, and hospitalization at the time of diagnosis was less frequent in the Vietnamese than in the German and Swiss patients $(78 \%$ versus $94 \%, p<0.005)$. The time of hospitalization during the first 6 mo after diagnosis differed between the two cohorts ( $p=$ $0.016)$. The percentage of patients hospitalized during the first 6 mo was $6 \%(1-5 d)$ and $16 \%(>5 d)$ in Vietnam, and 20\% $(1-5 \mathrm{~d})$ and $7 \%(>5 \mathrm{~d})$ in Germany and Switzerland. Initial treatment consisted of either corticosteroids $(84 \%)$ or no treatment $(16 \%)$ in the Vietnamese patients, and of IVIg $(80 \%)$, IVIg plus corticosteroids $(14 \%)$, or no treatment $(6 \%)$ in the German and Swiss cohort. There was no statistical difference in platelet counts at 6 mo between untreated and initially treated children in the two cohorts. When the subgroups acute and chronic ITP were evaluated, the only significant difference was the lower platelet counts in initially treated children with chronic ITP $\left(48 \pm 41 \times 10^{9} / \mathrm{L}\right.$ in the Vietnamese cohort, $n=$ $29)$, compared with the same group from the German and
Swiss cohort $\left(74 \pm 41 \times 10^{9} / \mathrm{L}, n=30 ; p=0.018\right)$. Additional treatment during the first 6 mo after the diagnosis of ITP, was given in Vietnam in 24 of 82 children $(29 \%)$ as corticosteroids, and in Germany and Switzerland in 20 of 71 children $(28 \%)$, as IVIg $(14 \%)$, corticosteroids $(6 \%)$, or IVIg plus corticosteroids $(8 \%)$.

\section{DISCUSSION}

Acute and chronic ITP are hemorrhagic disorders of unknown cause and pathogenesis occurring in both children and adults. The ITP phenotype occurring in two ethnically distinct cohorts living in different geographic regions was compared to ascertain whether environmental factors influenced the disease or, if differences in clinical management existed, which factors could also affect the course of the disease. This study revealed a number of important clinical and laboratory differences between the Vietnamese and the German and Swiss patients. It must be emphasized however, that these observations are to some extent based on subjective assessment and need to be confirmed. The age distribution described in the literature was observed only in the German and Swiss patients $(3,11)$. In Vietnam, more patients were found presenting at $<1$ y of age or $>10 \mathrm{y}$. This may represent a selection bias, although referral criteria were similar for both groups and the two cohorts as a whole were matched for age. The distribution of boys and girls was similar in both groups at the time of diagnosis.

Infection preceding the onset of ITP was more common in Germany and Switzerland. The association of infection with acute ITP is well described in the literature (12). Both the peak age and peak season (spring) of ITP diagnosis reflect the times of greatest susceptibility to various common viruses $(3,13)$, offering further support to this association. In our study, acute ITP occurred in equal numbers of children in both cohorts, although the incidence of infection was significantly different. The incidence of an infection preceding children with acute ITP and those who had chronic ITP was equally distributed (15\% and $17 \%$ in Vietnam, and $68 \%$ and $66 \%$ in Germany and Switzerland), suggesting a need for further studies investigating the role of infection in ITP.

Dry hemorrhage was seen more often in the Vietnamese cohort, and wet hemorrhage in Germany and Switzerland, but only among children with acute ITP. This observation is based on a very subjective measure and needs to be further investigated in large clinical trials. No such differences were evident between the two cohorts when patients with a platelet count $<20 \times 10^{9} / \mathrm{L}$ were considered. Hence, it seems likely that the differences in bleeding types between the two cohorts are explained by the platelet count. Quantification of bleeding is difficult and was not specifically investigated in our patients. In a recent study, the extent of bleeding was used as an important characterization of ITP in addition to the platelet count, emphasizing the importance of clinical aspects of ITP (14).

The initial platelet count was higher in Vietnamese patients compared with those from Germany and Switzerland. A selection bias might have contributed to this observation; however, every patient referred to each participating center, where the diagnosis of ITP was established, was eligible and met the 
standard inclusion criteria. Furthermore, the difference between the two patient cohorts remained statistically significant, even after exclusion of four Vietnamese patients who had relatively high initial platelet counts of $75-98 \times 10^{9} / \mathrm{L}$. This possible relationship of ethnic or environmental factors is analogous to that illustrated by the disease Onyalai, which may be a severe variant of ITP characterized by hemorrhagic bullae of the mucous membranes, particularly of the oropharynx, and associated with a high mortality. The disease has been described in the black population of central southern Africa, but not in the white population living in the same area (15).

In addition to the differences noted between the two cohorts, a number of important observations were made. A somewhat surprising observation was the unexpectedly high percentage of patients in both cohorts with the chronic form of ITP. The reason for this observation remains unclear. There were no predictors of the chronic form of the disease at the time of initial diagnosis. The severity of hemorrhage and the degree of thrombocytopenia, which may initiate referral of children with ITP, do not necessarily select for the chronic form of ITP. An estimate of the exact incidence of chronic ITP is difficult for many reasons, including inconsistent patient recruitment practices, lack of recognition of mild asymptomatic thrombocytopenia, heterogeneous etiological background of the disease, various patient ages, different management practices, and different definitions of chronic ITP. The impact of age on the incidence of chronic ITP is evident, supported by the estimation, that $47 \%$ of ITP patients aged $>14 \mathrm{y}$ have chronic ITP (16). George et al. (7) identified 12 uncontrolled case series with 1597 children followed for $\geq 12$ mo. ITP resolved in 1207 children $(76 \%)$, i.e. $24 \%$ had chronic ITP. Most of these case series were aimed at studying the effect of treatment on the course of ITP and used different definitions of chronic ITP, which was defined by the persistence of thrombocytopenia for 6 or 12 mo and with a platelet count of $<100$ or $<150 \times 10^{9} / \mathrm{L}$, and one study defined chronic ITP by the persistence of bleeding symptoms (5). The various definitions of chronic ITP of these mostly retrospective case series limit the value of the assessment of the incidence of chronic ITP in children. Therefore, a comparison of the incidence of chronic ITP in our study population with that in other populations is difficult and points to the need for prospective controlled clinical studies to determine the natural history of ITP, based on a clear definition of chronic ITP. A recently established Intercontinental Childhood ITP Registry evaluates prospective data for a large number of children worldwide to better understand the disease (17). Notwithstanding the above highlighted limitations, the finding of a predominance of boys with chronic ITP, both in the Vietnamese (19 of $35,54 \%$ ) and the German and Swiss cohort (20 of $30,67 \%)$, is in contrast to most of the literature, which suggests a predominance of girls $(3,9,18-20)$. In support of our findings, a sex distribution more biased toward boys has also been reported by others $(8,11,21)$.

The contribution of treatment to the course of the disease remains unclear because of a lack of prospective data. Our cohorts were managed differently, inasmuch as corticosteroids were used in Vietnam because IVIg was not available, and IVIg was the main therapeutic agent in Germany and Switzer- land. In spite of these different therapeutic modalities, the outcome and number of patients having chronic ITP were similar in both groups, suggesting no influence of the initial treatment or the environment on the natural history of the disease. Also, untreated patients revealed a similar outcome to that of treated patients after $6 \mathrm{mo}$, with no difference being noted between the two cohorts. This lack of therapeutic effect is supported by the report of Blanchette et al. (22) on outcome of ITP patients randomly assigned to receive IVIg, corticosteroids, or no therapy. Chronic ITP, defined as a platelet count of $<150 \times 10^{9} / \mathrm{L}$ for $>6 \mathrm{mo}$, occurred after treatment with IVIg in 2 of 18 children, after corticosteroids in 5 of 17 children, and in 3 of 16 children without therapy. In an earlier prospective study, $70 \%$ of all children recovered within 6 mo irrespective of corticosteroid treatment, with chronic ITP being defined as persistence of a platelet count of $<100 \times 10^{9} / \mathrm{L}$ for $>6 \mathrm{mo}$ (23). The observation that initially treated children, who had chronic ITP, had a higher platelet count at 6 mo in Germany and Switzerland than in Vietnam remains unclear. Whether the different treatment practices contributed to that difference needs to be investigated in larger clinical trials.

Although the comparative data between the Vietnamese and the German and Swiss children with ITP revealed similarities, important differences concerning the initial platelet count, the role of infection as a trigger of ITP, the type of bleeding, features of acute and chronic ITP, and management issues were found. Some of these findings are in contrast to the literature, such as the unexpected low occurrence of pre-ITP infection in Vietnamese children, and the high incidence of chronic ITP in both cohorts, and clearly justify further multicenter studies for confirmation.

\section{REFERENCES}

1. Parker Levine S 1999 Thrombocytopenia caused by immunologic platelet destruction In: Lee GR, Foerster J, Lukens J, Paraskevas F, Greer JP, Rodgers GM (eds) Wintrobe's Clinical Hematology, 10th Ed. Williams \& Wilkins, Baltimore, pp $1583-1611$

2. Schultz Beardsley D, Nathan DG 1998 Platelet abnormalities in infancy and childhood. In: Nathan DG, Orkin SH (eds) Nathan and Oski's Hematology of Infancy and Childhood, 5th Ed. WB Saunders, Philadelphia, pp 1585-1630

3. Lusher JM, Zuelzer WW 1966 Idiopathic thrombocytopenic purpura in childhood. J Pediatr 68:971-979

4. Nieminen U, Peltola H, Syrjala MT, Makipernaa A, Kekomaki R 1993 Acute thrombocytopenic purpura following measles, mumps and rubella vaccination. Acta Paediatr 82:267-270

5. Walker RW, Walker W 1984 Idiopathic thrombocytopenia, initial illness and long term follow up. Arch Dis Child 59:316-322

6. Rand ML, Wright JF 1998 Virus-associated idiopathic thrombocytopenic purpura. Transfus Sci 19:253-259

7. George JN, Woolf SH, Raskob GE, Wasser JS, Aledort LM, Ballem PJ, Blanchette VS, Bussel JB, Cines DB, Kelton JG, Lichtin AE, McMillan R, Okerbloom JA, Regan DH, Warrier I 1996 Idiopathic thrombocytopenic purpura: a practice guideline developed by explicit methods for The American Society of Hematology. Blood $88: 3-40$

8. Robb LG, Tiedeman K 1990 Idiopathic thrombocytopenic purpura: predictors of chronic disease. Arch Dis Child 65:502-506

9. Simons SM, Main CA, Yaish HM, Rutzky J 1975 Idiopathic thrombocytopenic purpura in children. J Pediatr 87:16-22

10. Lichtin A 1996 The ITP practice guideline: what, why, and for whom? Blood 88:1-2

11. Choi SI, McClure PD 1967 Idiopathic thrombocytopenic purpura in childhood. Can Med Assoc J 97:562-568

12. Waters AH 1992 Autoimmune thrombocytopenia: clinical aspects. Semin Hematol 29:18-25

13. Ferguson AW 1960 Rubella as a cause of thrombocytopenic purpura. Pediatrics 25:400-408

14. Medeiros D, Buchanan GR 1998 Major hemorrhage in children with idiopathic thrombocytopenic purpura: immediate response to therapy and long-term outcome. J Pediatr 133:334-339 
15. Hesseling PB 1987 Onyalai in Namibia: clinical manifestations, haematological findings, course and management of 103 patients in the Kavango territory. Trans R Soc Trop Med Hyg 81:193-196

16. Bottiger LE, Westerholm B 1972 Thrombocytopenia. I. Incidence and aetiology. Acta Med Scand 191:535-540

17. Kühne T 1999 Intercontinental childhood ITP registry. J Pediatr Hematol Oncol 21:308(abstr)

18. Aronis S, Platokouki H, Mitsiki A, Haidus S, Constantopoulos A 1994 Seventeen years of experience with chronic idiopathic thrombocytopenic purpura in childhood. Pediatr Hematol Oncol 11:487-498

19. Ramos ME, Newman AJ, Gross S 1978 Chronic thrombocytopenia in childhood. J Pediatr 92:584-586
20. Zaki M, Hassanein AA, Khalil AF 1990 Childhood idiopathic thrombocytopenic purpura: report of 60 cases from Kuwait. J Trop Pediatr 36:10-13

21. Yetgin S, Olcay L, Oezsoylu S, Hicsönmez G, Gürgey A, Tuncer AM 1997 Retrospective analysis of 78 children with chronic idiopathic thrombocytopenic purpura: follow-up from 1976 to 1996. Pediatr Hematol Oncol 14:399-412

22. Blanchette VS, Luke B, Andrew M, Sommerville-Nielsen S, Barnard D, de Veber B, Gent M 1993 A prospective, randomized trial of high-dose intravenous immune globulin $\mathrm{G}$ therapy, oral prednisone therapy, and no therapy in childhood acute immune thrombocytopenic purpura. J Pediatr123:989-995

23. Lammi AT, Lovric VA 1973 Idiopathic thrombocytopenic purpura: an epidemiologic study. J Pediatr 83:31-36 